\title{
Role of Macrophage Migration Inhibitory Factor (MIF) in Peripheral Nerve Regeneration: Anti-MIF Antibody Induces Delay of Nerve Regeneration and the Apoptosis of Schwann Cells
}

\author{
Yasuhiko Nishio, ${ }^{1}$ Jun Nishihira, ${ }^{2}$ Teruo Ishibashi, ${ }^{2}$ Hiroyuki Kato, ${ }^{1}$ and Akio Minami ${ }^{1}$ \\ ${ }^{1}$ Department of Orthopaedic Surgery \\ ${ }^{2}$ Department of Molecular Biochemistry, Hokkaido University Graduate School of Medicine, Sapporo, Japan \\ Accepted July 25, 2002
}

\begin{abstract}
Background: Macrophage migration inhibitory factor (MIF) is a pluripotent cytokine involved in inflammation and immune responses as well as in cell growth. Although we previously demonstrated the presence of MIF in peripheral nerves, and MIF mRNA expression was up-regulated after axotomy, the role of MIF in nerve injury and regeneration has not been evaluated.

Materials and Methods: To examine the potential role of MIF in nerve regeneration, we locally administered an anti-MIF polyclonal antibody into regenerating rat sciatic nerves using the silicone chamber model. The effect of the anti-MIF antibody on nerve regeneration was evaluated using an axonal reflex test. In addition, we carried out a terminal deoxynucleotidyl transferasemediated biotinylated UTP nick end labeling (TUNEL) assay and immunohistochemical analysis of the damaged nerve segments with regard to apoptosis-related proteins such as p53 to evaluate the effects of anti-
\end{abstract}

MIF antibodies on apoptosis during the regeneration process.

Results: The regeneration length of the nerve in the antiMIF antibody-treated group was significantly shorter than that in the non-immune rabbit IgG-treated group at weeks 2, 4 and 6 after surgery. TUNEL assay showed that a large number of apoptotic cells, mostly Schwann cells, were observed in the intratubal and distal nerve segments at weeks 4 and 6 after surgery by the anti-MIF antibody treatment. Consistent with these results, Ki-67-positive cells were significantly decreased by the anti-MIF antibody treatment. Immunohistochemical analyses revealed that p53 and, to a lesser extent, Fas were more up-regulated in the anti-MIF antibody-treated nerves than in the controls. Conclusion: Taken together, these results suggest that MIF plays an important role in acceleration of peripheral nerve regeneration and in prevention of Schwann cell apoptosis, mainly through overcoming the apoptotic effect of p53.

\section{Introduction}

The process of peripheral nerve degeneration and regeneration is a sequential event following nerve injury. Damage to peripheral nerves sufficient to separate a portion of the axon from the cell body leads to rapid degeneration of the isolated segment of the axon and the accompanying myelin sheath, which process is known as Wallerian degeneration (1). Distal to the site of transection, both the axon and its myelin sheath disintegrate and are digested by the Schwann cells, which proliferate and are able to act as facultative phagocytes similar to macrophages (2). Extensive Schwann cell mitosis occurs in the distal stump following nerve

Address correspondence and reprint requests to: J. Nishihira, Department of Molecular Biochemistry, Hokkaido University Graduate School of Medicine, Kita-15, Nishi-7, Kita-ku, Sapporo 060-8638, Japan. Tel/Fax: 81-11-706-5046; e-mail: j_nisihi@med.hokudai.ac.jp. injury, and actual cell numbers depend on the balance between proliferation and death. The factors that initiate Schwann cell mitosis and death in these situations have not yet been fully investigated, and may differ among various pathologic processes. Because axonal regrowth is dependent on the survival and proliferation of Schwann cells distal to the injury site, it is quite important to clarify the mechanism of these processes.

Macrophages play an important role in the nerve degeneration-regeneration process by producing cytokines and stimulating Schwann cells to proliferate and to play the role of phagocytes. The recruitment of macrophages from the peripheral vasculature is induced by chemoattractants released by infiltrating mononuclear cells. In the process of recruitment, macrophage migration inhibitory factor (MIF) is considered to play a pivotal role to concentrate macrophages at inflammatory loci $(3,4)$. To date, a number of studies have revealed that MIF is 
ubiquitously expressed in a variety of cells and tissues, including $\mathrm{T}$ cells and macrophages, and that it exerts broad-spectrum biological functions such as exacerbation of endotoxemia and T-cell activation (5-9). Recently, it has been indicated that MIF is potentially involved in cell proliferation and differentiation, including tumor-associated angiogenesis (10-15). In our previous reports, we found that MIF mRNA was up-regulated during tissue healing after corneal and epidermal injury $(16,17)$. In addition, we demonstrated the presence of MIF in the peripheral nervous system, where Schwann cells were the potential sources of MIF protein in peripheral nerves and MIF mRNA expression was up-regulated after axotomy (18). However, the precise pathophysiological functions of MIF in nerve regeneration remained to be elucidated.

In the present study, we locally administered antiMIF antibodies into regenerating rat sciatic nerves using the silicone chamber model, and the effect of antiMIF antibody administration on nerve regeneration was assessed using an axonal reflex test and antineurofilament immunohistochemical staining. Moreover, effects of anti-MIF antibodies on apoptosis were examined during the course of the degenerationregeneration process, during which immunohistochemical analyses on apoptosis-related proteins were carried out to evaluate their involvement.

\section{Material and Methods Materials}

The following materials were obtained from commercial sources: Tissue-Tek OCT compound from Miles Scientific (Naperville, IL); normal goat serum from Jackson (West Grove, PA); rabbit anti-bovine neurofilament polyclonal antibody (AB1981), rabbit antimouse p75 polyclonal antibody (AB1554), and rabbit anti-rat S-100 polyclonal antibody from Chemicon (Temecula, CA); fluorescein isthiocyanate (FITC)conjugated goat anti-rabbit immunoglobulin $\mathrm{G}$ from Zymed (San Francisco, CA); mouse anti-human Bcl-2 monoclonal antibody (sc7382) which cross-reacts with rat Bcl-2, rabbit anti-human $\mathrm{p} 53$ polyclonal antibody (sc6243), and rabbit anti-human Fas polyclonal antibody (sc7886) from Santa Cruz (Santa Cruz, CA); anti-CD3 antibody from Pharmingen (San Jose, CA); anti-macrophage antibody (ED2) from Serotec (Oxford, UK); anti-rat Ki-67 from DAKO (MIB-5) (Glostrup, Denmark); ApopTag ISOL kit from Intergen (Oxford, UK); Histofine SAB-PO kit and 3,3'-diaminobenzidine tetrahydrochloride from Nichirei (Tokyo, Japan); polyL-lysine from Sigma (St. Louis, MO); non-immune rabbit immunoglobulin G (IgG) from ICN (Costa Mesa, CA); terminal deoxynucleotidyl transferase (TdT) from Takara (Otsu, Japan); Protein A Sepharose from Pharmacia (Uppsala, Sweden); and proteinase K and biotin-16-2'-deoxyuridine-5' -triphosphate (biotin16-dUTP) from Roche (Indianapolis, IN). All other chemicals were of analytical grade.

\section{Preparation of MIF Antibodies}

Rat recombinant MIF was expressed in E.coli and purified to homogeneity as previously described (19). Polyclonal anti-rat MIF antiserum was generated by immunizing New Zealand White rabbits with purified recombinant MIF. In brief, the rabbits were inoculated intradermally with $100 \mu \mathrm{g}$ of MIF diluted in complete Freund's adjuvant at weeks 1 and 2, and with $50 \mu \mathrm{g}$ of MIF in incomplete Freund's adjuvant at week 4 . Immune serum was collected 1 week after the last inoculation. The IgG fraction $(4 \mathrm{mg} / \mathrm{ml})$ was purified with Protein A Sepharose according to the manufacturer's protocol. The specificity for rat MIF was confirmed by Western blot analysis as described (16).

\section{Animals}

All experiments were carried out on male 10-weekold Wistar King rats weighing around 350 gram. The animals were housed 3-5 per cage under controlled temperature and a fixed lighting schedule (lights on 06:00-18:00). All animal procedures were approved by the Regulations for Animal Experiments of the Institute for Animal Experimentation, Hokkaido University Graduate School of Medicine.

\section{Surgery}

The rats were anesthetized with sodium pentobarbital $(50 \mathrm{mg} / \mathrm{kg}$, i. p.), then the sciatic nerves were exposed bilaterally and transected midthigh level under aseptic conditions. Silicone tubes were made of medical-grade silicone tubing (internal diameter, $2.0 \mathrm{~mm}$; external diameter, $3.0 \mathrm{~mm}$; and length, $14 \mathrm{~mm})$. The internal diameter of the silicone tubes was chosen so as not to create any compression due to swelling of the enclosed nerve segment. The tubes were cut open with a longitudinal incision then wetsterilized. The silicone tube was sutured with a double epineurial stitch of 9-0 nylon to the divided ends of the sciatic nerve under a surgical microscope. Each nerve end was sutured $2 \mathrm{~mm}$ into the silicone chamber leaving a gap of $10 \mathrm{~mm}$ between the divided ends (Scheme). The chamber at the left leg was filled with $40 \mu \mathrm{l}$ non-immune rabbit IgG $(50 \mu \mathrm{g} / \mathrm{ml})$ as a control, and the chamber at the right was filled with $40 \mu \mathrm{l}$ anti-rat MIF antibodies $(50 \mu \mathrm{g} / \mathrm{ml})$. The skin wound was closed with 4-0 nylon sutures. Every three days, the animals were anesthetized and non-immune rabbit IgG or anti-rat MIF antibody was administered

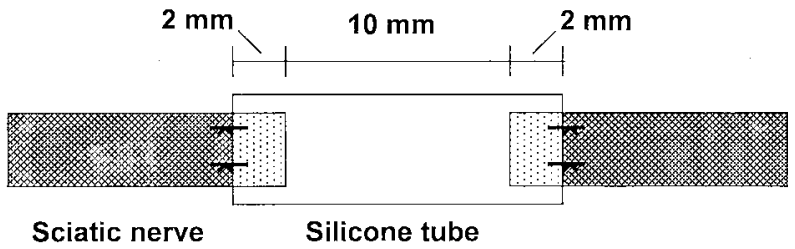

Scheme The silicone chamber model of the injured rat sciatic nerve. 
into the silicone chambers up until the time at which animals were sacrificed.

\section{Pinch Reflex Test}

At weeks 2, 4, and 6 after surgery, rats were anesthetized, and the sciatic nerves were re-exposed bilaterally and the silicone tubes removed. The sciatic nerve, including the peroneal and tibial nerves, was carefully dissected free distally, and cut at the level of the ankle. The leading axons were localized by pinching the nerve in a proximal direction with a pair of forceps until a withdrawal reflex was elicited, and marked with a ligature as described (20-22). The length between this point and the marked crush site was measured with a caliper and regarded as the length of regeneration of the sensory axons.

\section{Immunohistochemistry}

Immunohistochemical analysis of the neurofilament, which is a specific protein for axons, was carried out to examine the number of regenerating axons. Animals were anesthetized and perfused transcardially with $50 \mathrm{ml}$ of saline followed by $100 \mathrm{ml}$ of $4 \%$ paraformaldehyde in $0.1 \mathrm{M}$ sodium phosphate buffer ( $\mathrm{pH}$ 7.0). The distal, proximal, and intratubal nerve segments were removed and postfixed in fixative containing $20 \%$ sucrose overnight at $4^{\circ} \mathrm{C}$. Five-mm sections from each segment were cut from the tissues located $5-10 \mathrm{~mm}$ distal and proximal to the nerve stump and the center of the bridging nerve in the silicone tube. Sections were embedded in OCT compound and snap frozen in liquid nitrogen. Ten- $\mu \mathrm{m}$ thick frozen transverse sections were cut in a cryostat (Leica, model 1720), mounted on poly-L-lysine-coated slides, and airdried at room temperature. The slides were stored at $-20^{\circ} \mathrm{C}$ before immunostaining.

The sections were pre-incubated for $\mathrm{l} \mathrm{hr}$ with phosphate-buffered saline (PBS) containing 10\% normal goat serum and $1 \%$ bovine serum albumin to block non-specific staining. The rabbit anti-bovine neurofilament polyclonal antibody was applied to the sections at a dilution of 1:200, and the sections were incubated overnight at $4^{\circ} \mathrm{C}$. After three washes with PBS, the tissue specimens were incubated with FITC-labeled goat anti-rabbit IgG at a dilution of 1:100 for $1 \mathrm{hr}$ at room temperature. A confocal laser scanning microscope (Bio-rad MRC-1024, Hemel Hempstead, UK) was used to count the number of regenerating axons. Slides were photographed at $400 \times$ magnification, and the number of visible fluorescence-labeled axons were counted.

We also performed immunohistochemical analyses on apoptosis-related proteins, p53, Fas, Bcl-2, and $\mathrm{p} 75$. The slides were stained with the avidinbiotin-peroxidase complex procedure using a Histofine SAB-PO Kit according to the manufacturer's protocol. They were then incubated with biotinylated goat anti-rabbit IgG and streptavidin-biotin complex at room temperature for $30 \mathrm{~min}$. The reaction was developed in 3,3'-diaminobenzidine tetrahydrochloride (DAB) containing $0.01 \%$ hydrogen peroxide, and the samples were mounted with alkylacrylate. In a similar manner, immunohistochemical analyses were carried out to identify Schwann cells, macrophages, and $\mathrm{T}$ cells, using anti-S100, antimacrophage, and anti-CD3 antibodies, respectively. To identify proliferating cells, an antibody against $\mathrm{Ki}-67$ protein was used.

\section{Terminal Deoxynucleotidyl Transferase-mediated Biotinylated UTP Nick End Labeling (TUNEL) Assay}

We used the TUNEL assay to examine the extent of Schwann cell apoptosis. Sections were incubated in $20 \mu \mathrm{g} / \mathrm{ml}$ proteinase $\mathrm{K}$ solution for $20 \mathrm{~min}$ at room temperature and preequilibrated with TdT buffer (30 mM Trizma base, pH 7.2, $140 \mathrm{mM}$ sodium caccodylate, $1 \mathrm{mM}$ cobalt chloride) before the addition of TdT (18 U/coverslip) and biotinylated dUTP. As for the negative control, sections were treated with distilled water instead of TdT buffer. The sections were incubated at $37^{\circ} \mathrm{C}$ for $60 \mathrm{~min}$, and the reaction was terminated by incubating in buffer $(300 \mathrm{mM}$ sodium chloride, $30 \mathrm{mM}$ sodium citrate) for $30 \mathrm{~min}$ at room temperature. The sections were rinsed in PBS containing 10\% normal goat serum and $1 \%$ bovine serum albumin to block non-specific staining, then they were incubated with streptavidinbiotin complex using a Histofine SAB-PO Kit at room temperature for $30 \mathrm{~min}$. The reaction was developed in 3,3'-diaminobenzidine tetrahydrochloride containing $0.01 \%$ hydrogen peroxide, and the samples were mounted with alkylacryates.

\section{Statistics}

Statistical comparison between groups was carried out using Student's t test.

\section{Results}

Pinch Reflex Test

The length of axonal outgrowth was measured at weeks 2, 4, and 6 after sacrifice. The regenerated axonal length of the anti-MIF antibody-treated groups was significantly shorter than that of the control at all time points (Fig. 1). At week 2 after surgery, the regeneration lengths of non-immune IgG-treated (control) and the anti-MIF antibody-treated groups were $4.3 \pm 1.4 \mathrm{~mm}$ and $1.9 \pm 1.4 \mathrm{~mm}$ (mean $\pm \mathrm{SD}$ ), respectively. The regeneration lengths of the control and anti-MIF antibody-treated groups at week 4 were $27.0 \pm 7.6 \mathrm{~mm}$ and $19.0 \pm 8.1 \mathrm{~mm}$, respectively, and $46.5 \pm 12.1 \mathrm{~mm}$ and $38.9 \pm 5.1 \mathrm{~mm}$, respectively, at week 6 . These results indicated that administration of the anti-MIF antibody could inversely affect regeneration after peripheral nerve injury.

\section{Neurofilament Staining}

To evaluate the number of regenerating axons, immunohistochemical analysis of neurofilaments was 


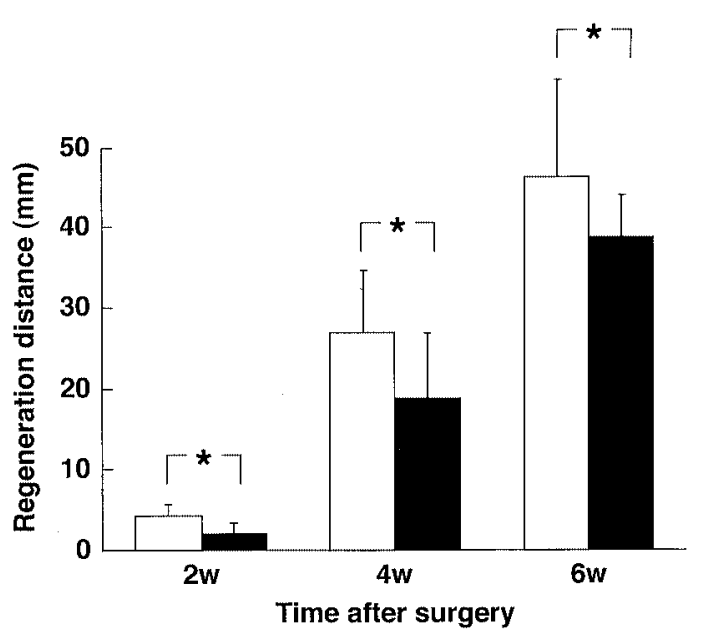

Fig. 1. Effect of anti-MIF antibody on peripheral nerve regeneration. Regeneration length of the injured peripheral nerve was measured by a pinch reflex test as described in "Materials and Methods." After sacrifice at the indicated intervals, regeneration length was measured. Regeneration length in the anti-MIF antibody-treated group was significantly shorter than that in the control group at weeks 2,4 , and 6 after surgery $(n=5$ for each week) ( $\left.{ }^{*} \mathrm{p}<0.01\right)$. Open bars, non-immune IgG; Closed bars, anti-MIF antibody. The values are expressed as mean \pm SD.

carried out. In the newly regenerated nerve segment in the silicone chamber, we found a significant decrease in the number of axons in the anti-MIF antibody-treated group $(640 \pm 318 ;$ mean \pm SD) compared to the number in controls $(1364 \pm 545)$ at week 2 after surgery (Fig. 2A). Similarly, we also observed a significant decrease of the number of axons in the anti-MIF antibody-treated group (2045 \pm 1270) compared to controls $(4000 \pm 1045)$ at week 4 after surgery, but the difference was not significant at week 6 .

\section{A}

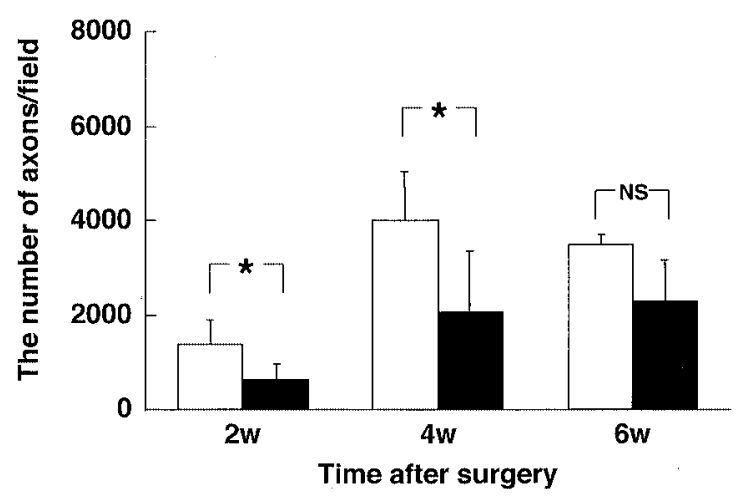

In the distal segment of transection, the number of neurofilament-positive axons in anti-MIF antibodytreated nerves $(820 \pm 410)$ was significantly less than that in the control nerves $(3140 \pm 910)$ at week 4 after surgery (Fig. 2B). However, no significant difference was observed between the number of regenerative axons in control nerves and that in anti-MIF antibody-treated nerves at weeks 2 and 6 after surgery. The slight difference of the distal nerve segments at week 2 might have been due to the fact that regenerated axons did not reach the distal segment.

TUNEL Assays

We examined sections of the rat sciatic nerve at weeks 4 and 6 after surgery, when regenerating axons had reached the distal nerve stump, using the TUNEL assay to identify the nuclei of cells undergoing DNA fragmentation characteristic of apoptosis. After nerve injury, Schwann cells remain the predominant cell type, even though fibroblasts and macrophages may also proliferate (23). Thus, it is considered that the majority of apoptotic nuclei in the sciatic nerve represent Schwann cells. The density of apoptotic cells was very low in both the intratubal (Fig. 3a, e) and distal segments (Fig. 3c, g) of the regenerating nerves treated with non-immune IgG at weeks 4 and 6 after surgery. On the other hand, a large number of apoptotic nuclei were observed in the intratubal segments treated with the anti-MIF antibody at weeks 4 and 6 after surgery (Fig. 3b, f). Similarly, high density of apoptotic nuclei was seen in the distal nerve segments treated with the anti-MIF antibody at week 6 after surgery (Fig. $3 \mathrm{~h})$, though the density was relatively low at week 4 (Fig. $3 \mathrm{~d}$ ). In the proximal segment of the control and the anti-MIF-treated sciatic nerves at all time points, TUNEL-positive cells were scarcely seen (data not shown).

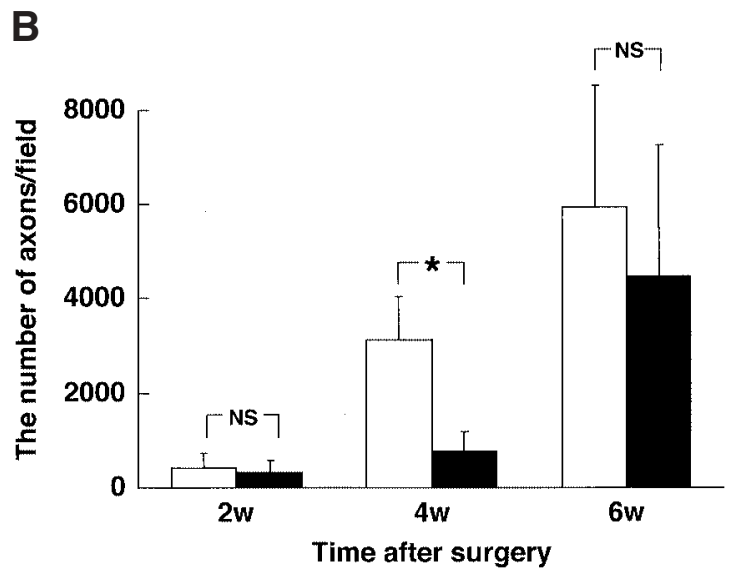

Fig. 2. Time-course study on regenerating axons. The numbers of regenerating axons were counted based on positive staining of neurofilaments per field $(\times 400)$. Anti-MIF antibodies were administered into regenerating rat sciatic nerves using the silicon chamber model as described in "Materials and Methods." (A) The number of axons in the silicon chamber. A significant difference was found between the anti-MIF antibody-treated and non-immune IgG-treated (control) nerve segments at weeks 2 and 4 after surgery ( $\mathrm{n}=5$ ) $\left({ }^{*} \mathrm{p}<0.05\right)$. Open bars, non-immune IgG; closed bars, anti-MIF antibody. (B) The number of axons in the distal nerve segment. A significant difference was found between the anti-MIF antibody-treated nerves and the control at week 4 after surgery $(n=5)\left({ }^{*} p<0.05\right)$. NS; not significant. Open bars, non-immune IgG; Closed bars, anti-MIF antibody. The values are expressed as mean \pm SD. 

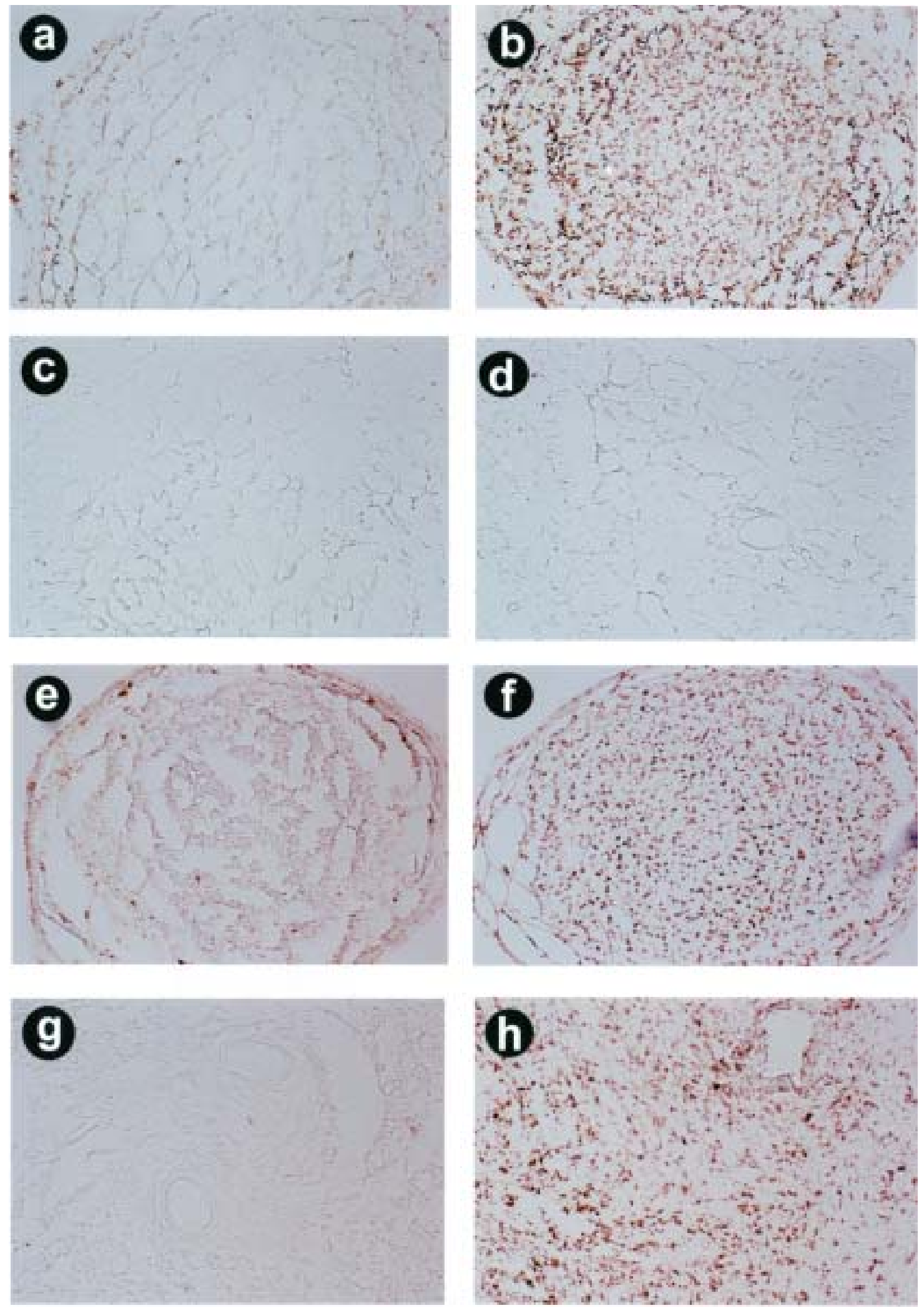

Fig. 3. Effect of anti-MIF antibody with regard to apoptosis after nerve damage. Apoptosis was assessed based on the nuclei of cells undergoing characteristic DNA fragmentation, using TUNEL assays. From (a) to (d) at 4 week after surgery. Intratubal nerve segments in the control group (a) and the anti-MIF antibody-treated group (b). Distal nerve segments in the control (c) and the anti-MIF antibody-treated group (d). From (e) to (h) at 6 week after surgery. Intratubal nerve segments in the control (e) and the anti-MIF antibody-treated group (f). Distal nerve segments in the control (g) and the anti-MIF antibody-treated group (h). All magnifications are $\times 100$. 


\section{Immunohistochemical Analysis of Apoptosis-related Proteins in the Intratubal Nerve Segments}

Immunohistochemical analyses on Fas and p53 were carried out for the intratubal nerve segments at weeks 4 and 6 after surgery, when a marked difference in the density of TUNEL-positive nuclei could be observed between anti-MIF antibody-treated and non-immune IgG-treated control groups. At week 4 after surgery, immunoreactivities of Fas and p53 for the intratubal nerve segments in controls treated with non-immune IgG were weak (Fig. 4a, c), whereas both proteins, particularly p53, were up-regulated in the anti-MIF antibodytreated tissue sections (Fig. 4b, d). The up-regulation of Fas and p53 by the anti-MIF antibody treatment was also found at week 6, but was less significant in comparison with those at week 4 (Fig. $4 \mathrm{f}, \mathrm{h}$ ). We also examined immunoreactivities of Bcl-2 and p75. We could not observe any significant relationship between MIF suppression and Bcl-2 or p75 (data not shown).

\section{Immunohistochemical Analysis of Apoptosis-related} Proteins in the Distal Nerve Segments

Immunohistochemical analyses of Fas and p 53 were also carried out for the distal nerve segments at weeks 4 and 6 after surgery in anti-MIF antibodytreated and control groups. At week 4 after surgery, immunoreactivity of p53 was markedly enhanced by anti-MIF antibody treatment compared with the control (Fig. 5c, d), but only a minimal increase was observed for Fas (Fig. 5a, b).

At week 6 after surgery, only slight up-regulation of immunoreactivity of p53 and Fas was observed in response to anti-MIF antibody treatment (Fig. 5, e-h). In addition, we performed immunohistochemistry using an Apoptag ISOL kit, which showed that most cells became apoptotic (data not shown). As for p75, it should be noted that its expression was clearly seen at week 4, but not at week 6 after surgery (data not shown). We could not observe any significant relationship between MIF suppression and Bcl-2, similar to the case of the intratubal segment.

\section{Immunohistochemistry for Schwann Cells}

To investigate cell populations, we first performed immunohistochemical analysis using an antibody against S-100 protein for Schwann cells. In the intratubal segments at week 4 , we found a number of S-100-positive cells in both non-immune IgG-treated (Fig. 6a) and anti-MIF antibody-treated sections (Fig. 6b). In the distal nerve segments, most of the observed cells were identified as S-100-positive cells in both control (Fig. 6c) and anti-MIF antibody-treated sections (Fig. 6d). Similar results were also obtained for week 6 after surgery (data not shown). No specific immunostaining was observed when non-immune IgG was used instead of the anti-S-100 antibody.

\section{Immunohistochemistry for T-cells and Macrophages}

As for $\mathrm{T}$ cells and macrophages, we carried out immunohistochemistry using an anti-CD3 antibody and anti-macrophage antibody, respectively, at week 4 after surgery. We could hardly observe CD-3-positive cells in the intratubal segment of the control (Fig. 7a) and anti-MIF antibody-treated groups (Fig. 7b). As for the distal nerve segment, few anti-CD3-positive cells were found in either control or anti-MIF antibody-treated sections (Fig. 7e, f).

We found anti-macrophage antibody-positive cells in intratubal (Fig. 7c) and distal nerve (Fig. 7g) segments treated with the non-immune IgG. It is of note that these positive cells were significantly decreased by the anti-MIF antibody treatment (Fig. 7d, h). Similar results were obtained for week 6.

\section{Cell Proliferation in the Intratubal and Distal Nerve Segments}

To examine the effect of the anti-MIF antibody on cell proliferation, we performed immunohistochemistry using an antibody against Ki-67. At week 4 after surgery, we observed Ki-67-positive cells in both intratubal (Fig. 8a) and distal nerve (Fig. 8c) segments in the controls, but the numbers of these positive cells were significantly suppressed by the anti-MIF antibody treatment (Fig. 8b, d). A similar reduction in the number of macrophages was obtained at week 6 (data not shown).

\section{Discussion}

It has been well demonstrated that the silicone chamber model is an excellent system for studying various aspects of peripheral nerve regeneration. The overall pattern of regeneration observed in our experiments was consistent with that in previous reports using comparable entubulation systems (24). Following transection and introduction of proximal and distal nerve stumps into the tube, a fibrin matrix developed between the nerve ends. The fibrin matrix was invaded by macrophages and, later, by ingrowth of Schwann cells and axons. In this context, the silicone chamber model has been used to evaluate the events occurring at the site of peripheral nerve repair as well as to analyze the efficacy of various neurotrophic factors.

Recently, it has been reported that proinflammatory cytokines such as interleukin (IL)-1 (25), IL-6 $(26,27)$, and tumor necrosis factor (TNF)- $\alpha$ $(28,29)$ play critical roles in the peripheral nerve degeneration-regeneration process. In particular, it is considered that IL-1 may be essential for upregulation of nerve growth factor (NGF) synthesis and thymocyte proliferation $(30,31)$. We previously reported the expression of MIF in peripheral nerves, and showed that Schwann cells were the potential source of MIF protein (18). Based on the indication that MIF functions to recruit macrophages to the inflammatory site, and is also involved in cell differentiation (10), we hypothesized that MIF plays an important role during the regeneration process of damaged peripheral nerves. 

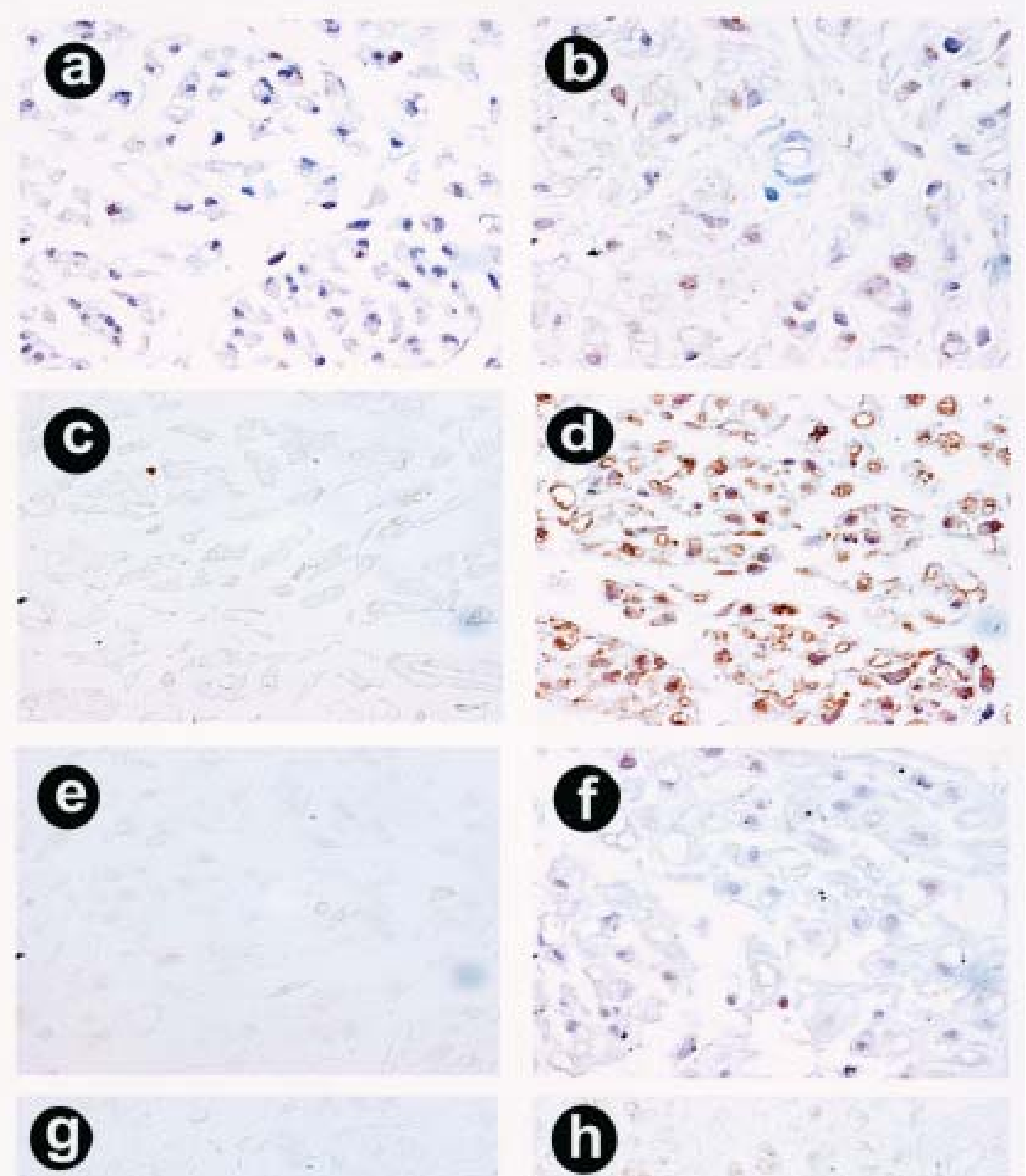

Fig. 4. Immunohistochemistry for apoptosis-related proteins in the intratubal nerve segment after damage. The immunohistochemical analysis was carried out using antibodies against apoptosis-related proteins in the intratubal nerve segments. From (a) to (d) at week 4 after surgery. Reacted with non-immune IgG (a) and anti-Fas antibody (b). Reacted with non-immune IgG (c) and anti-p53 antibody (d). From (e) to (h) at week 6 after surgy. Reacted with non-immune IgG (e) and anti-Fas antibody (f). Reacted with non-immune IgG (g) and anti-p53 antibody (h). All magnifications are $\times 400$. 

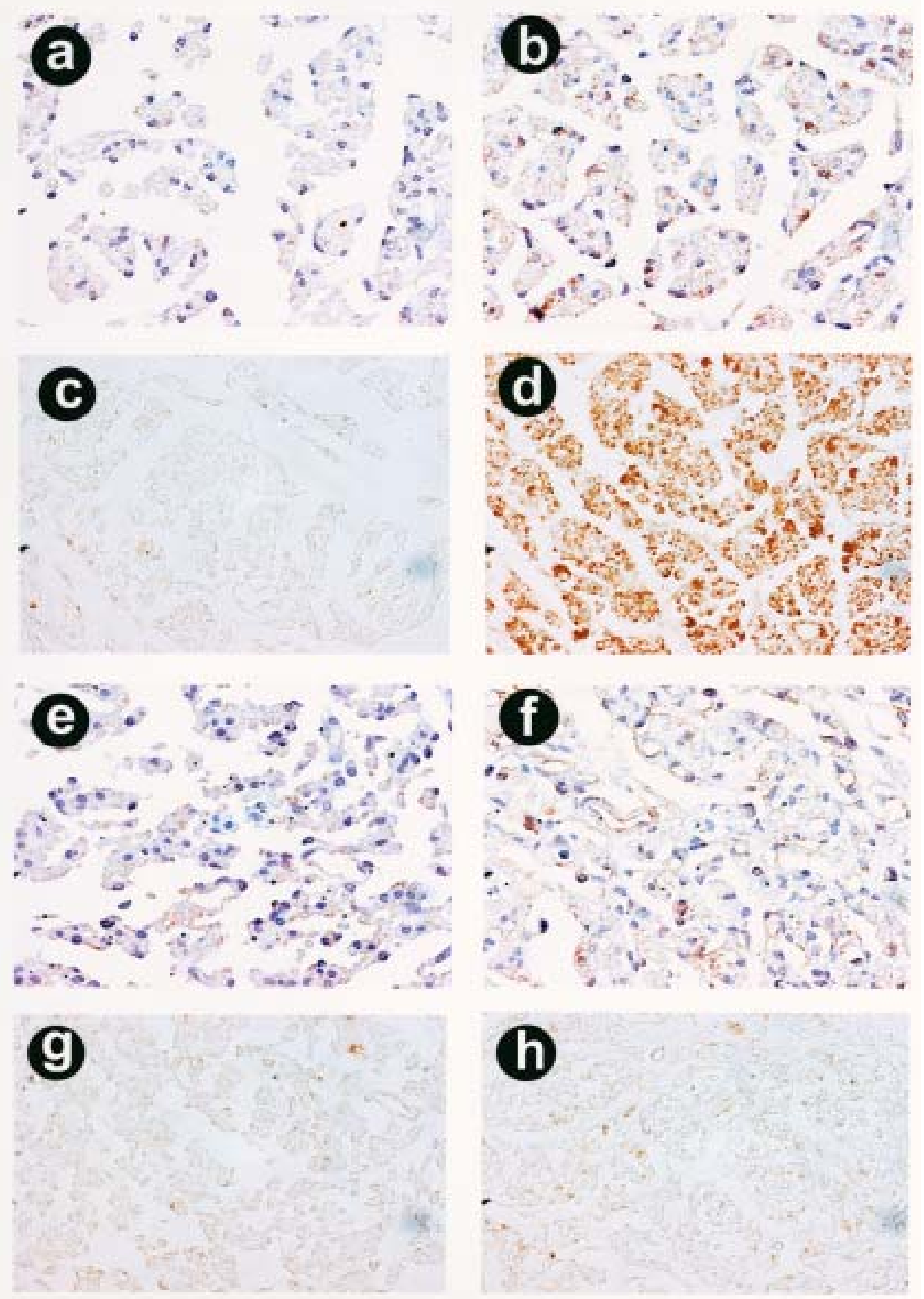

Fig. 5. Immunohistochemistry for apoptosis-related proteins in the distal nerve segments after nerve damage. The immunohistochemical analysis was carried out using antibodies against apoptosis-related proteins in the distal nerve segment. From (a) to (d) at week 4 after surgery. Reacted with non-immune IgG (a) and anti-Fas antibody (b). Reacted with non-immune IgG (c) and anti-p53 antibody (d). From (e) to (h) at week 6 after surgery. Reacted with non-immune IgG (e) and anti-Fas antibody (f). Reacted with nonimmune IgG (g) and anti-p53 antibody (h). All magnifications are $\times 400$. 

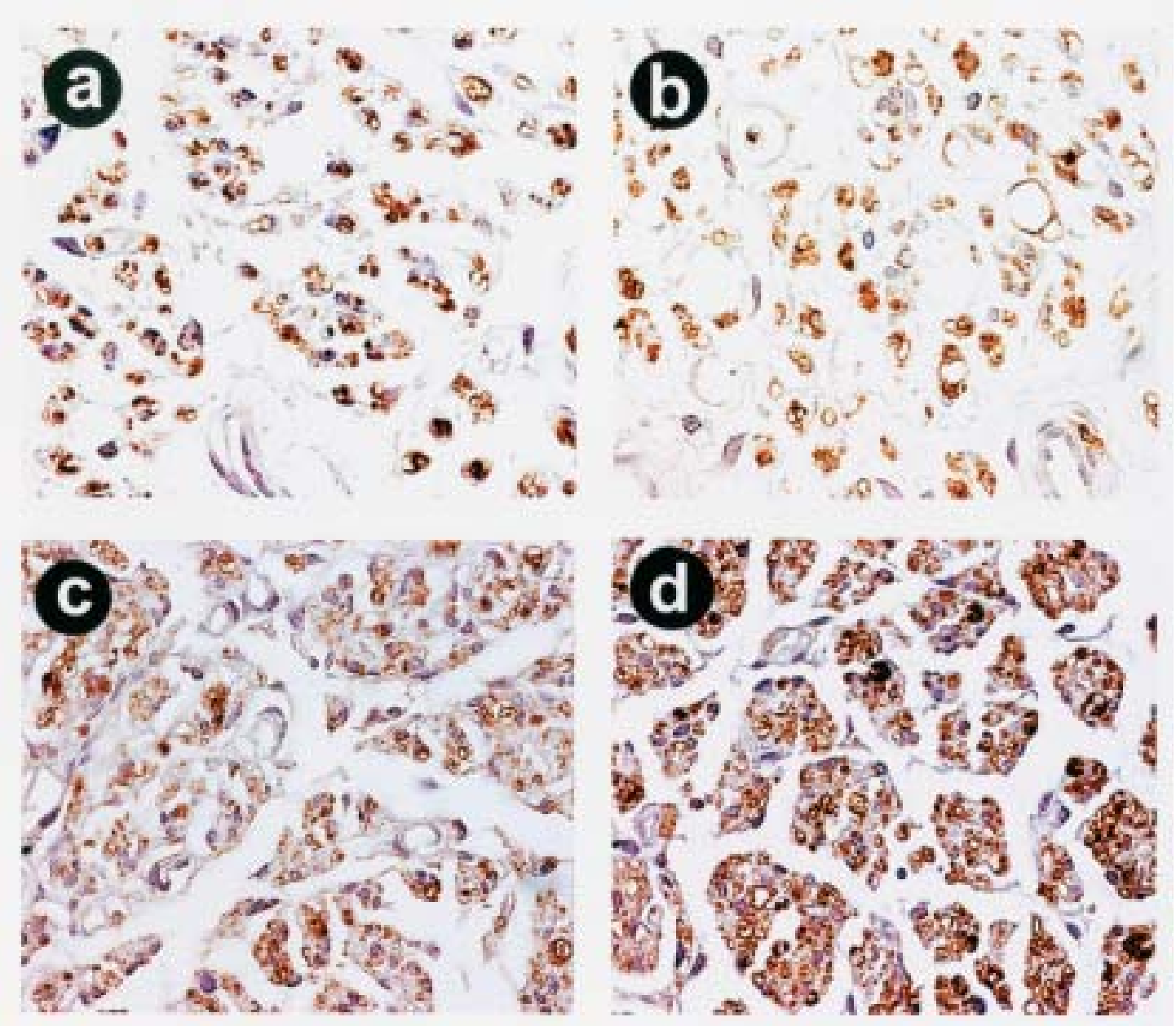

Fig. 6. Immunohistochemistry for Schwann cells in intratubal and distal nerve segments. The immunohistochemical analysis was carried out on tissue specimens from intratubal and distal nerve segments treated with the anti-MIF antibody, which were obtained at week 4 after surgery using an antibody against S-100 protein. Reacted with non-immune rabbit IgG (a) and anti-S100 antibody (b) for intratubal nerve segments. Reacted with the non-immune IgG (c) and anti-S100 antibody (d) for distal nerve segments. No positive staining was observed when non-immune rabbit IgG was used instead of the anti-S-100 antibody, and similar results were obtained for the tissue sections obtained at week 6 . All magnifications are $\times 400$.

We administered anti-MIF antibodies into the silicone chambers to assess the hypothesis that suppression of MIF could hamper nerve regeneration. As shown in this study, administration of the anti-MIF antibody led to a delay of peripheral nerve regeneration. In a previous study, we showed that MIF mRNA expression was up-regulated after nerve transection, but decreased within three weeks after nerve injury (18). In this context, it is conceivable that MIF may exert its stimulant effect on nerve regeneration within four weeks after axotomy. It is considered that Schwann cells and, to a lesser extent, macrophages are the major sources of MIF based on the results of immunohistochemistry. Taken together, these findings indicate that MIF may play a pivotal role in the early stages of the peripheral nerve degenerationregeneration process.

Furthermore, we demonstrated that MIF convincingly affected the survival of Schwann cells. The
TUNEL assay revealed that a large number of Schwann cells underwent apoptosis after anti-MIF antibody administration, during the process of nerve regeneration. Extensive Schwann cell mitosis occurs in the distal nerve stump following nerve injury, but, as is the case with other proliferative tissues, actual cell numbers depend on the balance between cell proliferation and death. In adult rodents, it is well established that Schwann cell apoptosis does not occur in the distal stump of axotomized nerves even 60 days after axotomy (32). Therefore, it was likely that apoptosis would be scarcely identified in the axotomized nerves of controls at weeks 4 and 6 after surgery. However, marked apoptosis of Schwann cells was detected in the anti-MIF antibody-treated axotomized nerves. These results suggest the possibility that suppression of MIF results in Schwann cell apoptosis. Thus, MIF may have a function to protect Schwann cells from apoptosis, and to maintain Schwann cell 

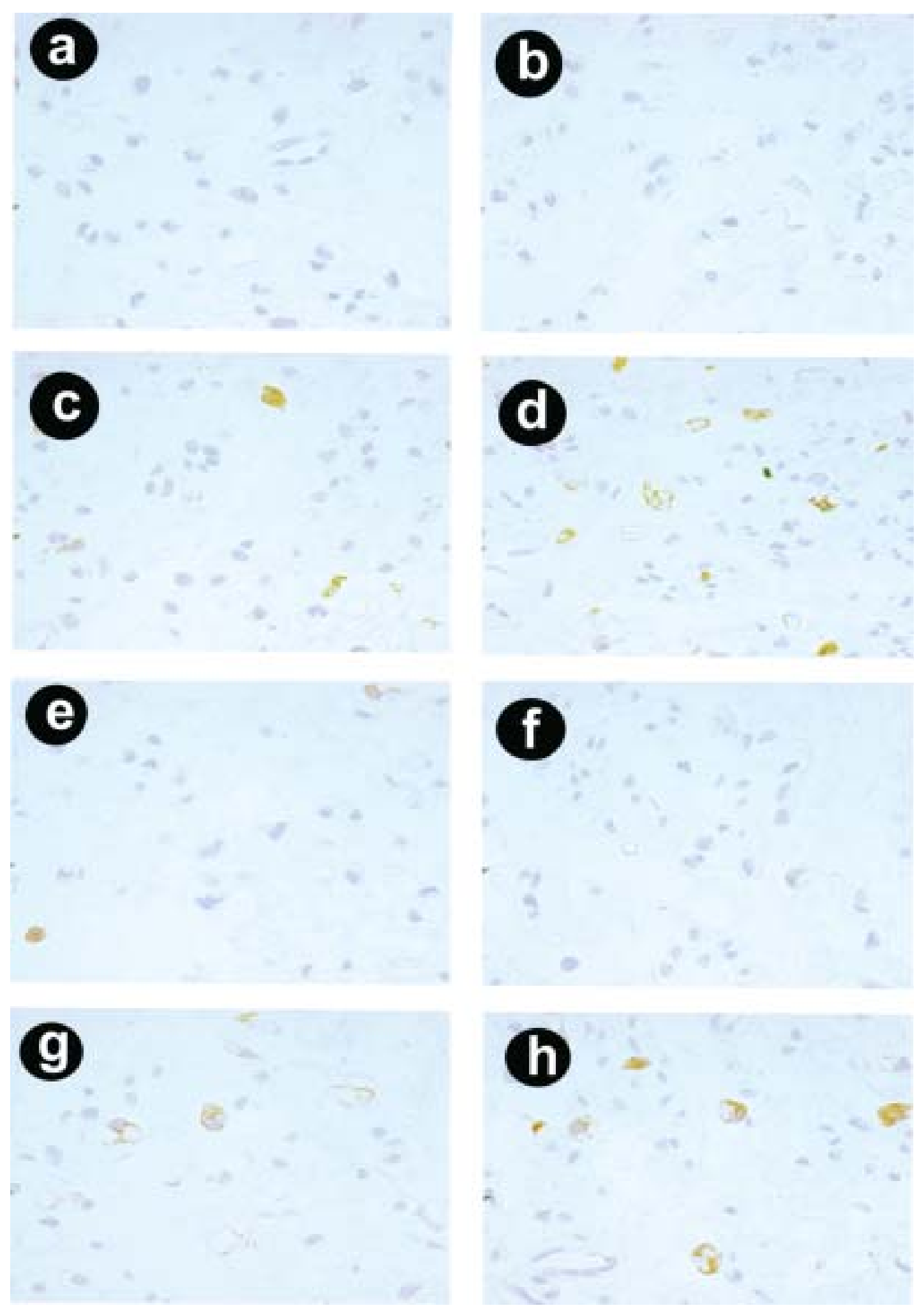

Fig. 7. Immunohistochemistry for lymphocytes and macrophages in the intratubal and distal nerve segments at 4 weeks after surgery. The immunohistochemical analysis was carried out using an anti-CD3 antibody and anti-macrophage antibody (ED2). From (a) to (d), the results of intratubal nerve segments are shown. Reacted with anti-CD3 antibody after treatment with non-immune IgG (a) and anti-MIF antibody (b). Reacted with anti-macrophage antibody after treatment with non-immune IgG (c) and anti-MIF antibody (d). From (e) to (h), the results of distal nerve segments are shown. Reacted with anti-CD3 antibody after treatment with non-immune IgG (e) and anti-MIF antibody (f). Reacted with anti-macrophage antibody after treatment with non-immune IgG (g) and anti-MIF antibody (h). All magnifications are $\times 400$. 


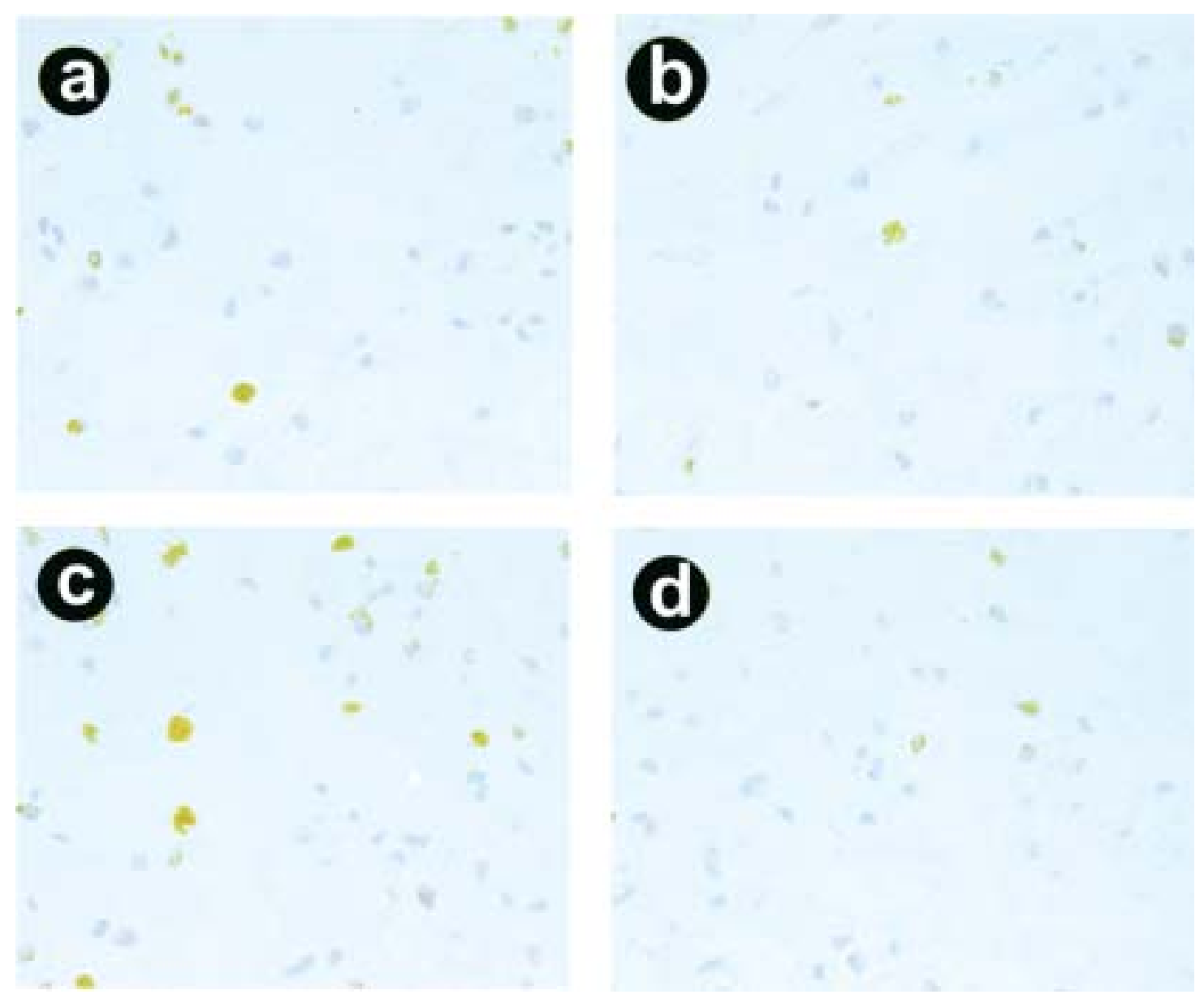

Fig. 8. Immunohistochemistry for Ki-67 protein with regard to cell proliferation in the intratubal and distal nerve segments. The immunohistochemical analysis was carried out using the antibody against Ki-67 protein at week 4 after surgery. Intratubal segments treated with non-immune IgG (a) and anti-MIF antibody (b). Distal nerve segments treated with non-immune IgG (c) and anti-MIF antibody (d). All magnifications are $\times 400$. Similar results were obtained for the tissue samples obtained at week 6 .

viability. Consistent with these findings, we found that cell proliferation was significantly suppressed by the anti-MIF antibody as demonstrated by immunohistochemical analysis using proliferation-associated protein Ki-67.

We demonstrated expression levels of apoptosisrelated proteins in the event of Schwann cell apoptosis in response to anti-MIF antibody administration using immunohistochemical analysis. At weeks 4 and 6 after surgery, we found that p53 and Fas were more immunoreactive in the anti-MIF antibody-treated nerves than in the controls. Based on these facts, it appears that apoptosis in response to $\mathrm{p} 53$ or Fas activation might be induced by the continuous suppression of MIF functions by the anti-MIF antibody. Concerning the molecular mechanism of apoptosis in association with MIF, it was first reported that MIF has the potential to inhibit the biologic action of a tumor suppressor oncogene, p53, which plays a key role in cell proliferation and apoptosis (33).

Subsequently, it was revealed that MIF could sustain macrophage survival and function by suppressing p53-induced apoptosis based on results from MIFdeficient mice (34). With regard to the molecular mechanism, it was demonstrated that MIF inhibits p53 activity in macrophages via an autocrine regulatory pathway, which results in a decrease in cellular p53 accumulation and subsequent function. Based on these findings, including ours, it is considered that MIF may play an anti-apoptotic role for Schwann cells, possibly through inhibition of p53 bioactivity.

We could not observe any significant relationship between MIF suppression and $\mathrm{Bcl}-2$ regulation in either intratubal or distal nerve segments; however, p75 expression was clearly seen in the distal nerve segment at week 4 after surgery, but scarcely found at week 6 (data not shown). It was reported that upregulation of p75 can induce apoptosis through NGF activation (35). In addition, it has been demonstrated that axotomy leads to potentiated apoptosis among Schwann cells deprived of axonal contact $(32,36)$, and that the increase of p75 expression reaches a maximum one month after nerve injury (37). In this context, the pathway inducing apoptosis of Schwann cells 
by the anti-MIF antibody in the event of peripheral nerve injury may involve not only p53, but also $\mathrm{p} 75$.

Finally, we found that administration of the antiMIF antibody to damaged nerve tissues induced a delay of regeneration, decrease of axons, and marked apoptosis of Schwann cells. These data strongly suggest that MIF plays a key role not only in peripheral nerve regeneration, but also in viability of Schwann cells by the suppression of apoptosis-related proteins such as p53. The current study may shed light on the novel role of MIF in the nervous system, and could augur therapeutic application of this cytokine to nerve damage.

\section{Acknowledgments}

We are grateful to $\mathrm{S}$. Tone for her technical assistance, and to Y. Mizue, of the Sapporo Immunodiagnostic Laboratory, for preparing the anti-rat MIF antibody.

\section{References}

1. Holmes W, Young JZ. (1942) Nerve regeneration after immediate and delayed suture. $J$ Anat 77: 63-96.

2. Perry VH, Brown MC. (1992) Role of macrophages in peripheral nerve degeneration and repair. Bioessays 14: 401-406.

3. David JR. (1966) Delayed hypersensitivity in vitro: its mediation by cell free substances forms by lymphoid cell-antigen interaction. Proc. Natl. Acad. Sci. USA 56: 72-77.

4. Bloom BR, Bennett B. (1966) Mechanism of a reaction in vitro associated with delayed-type hypersensitivity. Science 153: $80-82$.

5. Bernhagen J, Calandra T, Mitchell RA, et al. (1993) MIF is a pituitary-derived cytokine that potentiates lethal endotoxemia. Nature 365: 756-759.

6. Calandra T, Bernhagen J, Metz CN, et al. (1995) MIF as a glucocorticoid-induced modulator of cytokine production. Nature 377: 68-71.

7. Bacher M, Metz CN, Calandra T, et al. (1996) An essential regulatory role for macrophage migration inhibitory factor in T-cell activation. Proc. Natl. Acad. Sci. USA 93: 7849-7854.

8. Calandra T, Bernhagen J, Mitchell RA, Bucala R. (1994) The macrophage is an important and previously unrecognized source of macrophage migration inhibitory factor. J. Exp. Med. 179: 1895-1902.

9. Bucala R. (1996) MIF re-discovered: pituitary hormone and glucocorticoid-induced regulator of cytokine production. FASEB J. 7: 19-24.

10. Wistow GJ, Shaughnessy MP, Lee DC, et al. (1993) A macrophage migration inhibitory factor is expressed in the differentiating cells of the eye lens. Proc. Natl. Acad. Sci. USA 90: 1272-1275.

11. Suzuki H, Kanagawa H, Nishihira J. (1996) Evidence for the presence of macrophage migration inhibitory factor in murine reproductive organs and early embryos. Immunol. Lett. 51: 141-147.

12. Mitchell RA, Metz CN, Peng T, Bucala, R. (1999) Sustained mitogen-activated protein kinase (MAPK) and cytoplasmic phospholipase A2 activation by macrophage migration inhibitory factor (MIF): regulatory role in cell proliferation and glucocorticoid action. J. Biol. Chem. 274: 18100-18106.

13. Chesney J, Metz C, Bacher M, et al. (1999) An essential role for macrophage migration inhibitory factor (MIF) in angiogenesis and the growth of a murine lymphoma. Mol. Med. 5: 181-191.

14. Shimizu T, Abe R, Nakamura H, et al. (1999) High expression of macrophage migration inhibitory factor in human melanoma cells and its role in tumor cell growth and angiogenesis. Biochem. Biophys. Res. Commun. 264: 75 1-758.

15. Ogawa H, Nishihira J, Sato Y, et al. (2000) An antibody for macrophage migration inhibitory factor suppresses tumor growth and inhibits tumor-associated angiogenesis. Cytokine 12: 309-314.

16. Abe R, Shimizu T, Ohkawara A, Nishihira J. (2000) Enhancement of macrophage migration inhibitory factor (MIF) expression in injured epidermis and cultured fibroblasts. Biochim. Biophys. Acta 1500: 1-9.

17. Matsuda A, Tagawa Y, Matsuda H, Nishihira J. (1997) Expression of macrophage migration inhibitory factor in corneal wound healing in rats. Invest. Ophthalmol. Vis. Sci. 38: 1555-1562.

18. Nishio $\mathrm{Y}$, Minami A, Kato $\mathrm{H}$, et al. (1999) Identification of macrophage migration inhibitory factor (MIF) in rat peripheral nerves: its possible involvement in nerve regeneration. Biochim. Biophys. Acta 1453: 74-82.

19. Nishihira J, Kuriyama T, Sakai M, et al. (1995) The structure and physicochemical properties of rat liver macrophage migration inhibitory factor. Biochim. Biophys. Acta 1247: 159-162.

20. Kanje M, Lundborg G, Edstrom A. (1988) A new method for studies of the effects of locally applied drugs on peripheral nerve regeneration in vivo. Brain Res. 439: 116-121.

21. Kerns JM, Danielsen N, Holmquist B, et al. (1993) The influence of predegeneration on regeneration through peripheral nerve grafts in the rat. Exp. Neurol. 122: 28-36.

22. Danielsson P, Dahlin L, Povlsen B. (1996) Tubulization increases axonal outgrowth of rat sciatic nerve after crush injury. Exp. Neurol. 139: 238-243.

23. Ferri CC, Bisby MA. (1999) Improved survival of injured sciatic nerve Schwann cells in mice lacking the p75 receptor. Neurosci. Lett. 272: 191-194.

24. Williams LR, Longo FM, Powell HC, et al. (1983) Spatialtemporal progress of peripheral nerve regeneration within a silicone chamber: parameters for a bioassay. J. Comp. Neurol. 218: $460-470$.

25. Korompilias AV, Chen LE, Seaber AV, Urbaniak JR. (1999) Interleukin-1 beta promotes functional recovery of crushed peripheral nerve. J. Orthop. Res. 17: 714-719.

26. Bolin LM, Verity AN, Silver JE, et al. (1995) Interleukin-6 production by Schwann cells and induction in sciatic nerve injury. J. Neurochem. 64: 850-858.

27. Bourde O, Kiefer R, Toyka KV, Hartung HP. (1996) Quantification of interleukin- 6 mRNA in wallerian degeneration by competitive reverse transcription polymerase chain reaction. J. Neuroimmunol. 69: 135-140.

28. Chen LE, Seaber AV, Wong GH, Urbaniak JR. (1996) Tumor necrosis factor promotes motor functional recovery in crushed peripheral nerve. Neurochem. Int. 29: 197-203.

29. Wagner R, Myers RR. (1996) Schwann cells produce tumor necrosis factor alpha: expression in injured and non-injured nerves. Neuroscience 73: 625-629.

30. Lindholm D, Heumann R, Meyer M, Thoenen H. (1987) Interleukin-1 regulates synthesis of nerve growth factor in non-neuronal cells of rat sciatic nerve. Nature 330: 658-659.

31. Rotshenker S, Aamar S, Barak V. (1992) Interleukin-1 activity in lesioned peripheral nerve. J. Neuroimmuno.l 39: 75-80.

32. Grinspan JB, Marchionni MA, Reeves M, et al. (1996) Axonal interactions regulate Schwann cell apoptosis in developing peripheral nerve: neuregulin receptors and the role of neuregulins. J. Neurosci. 16: 6107-6118.

33. Hudson JD, Shoaibi MA, Maestro R, et al. (1999) A proinflammatory cytokine inhibits p53 tumor suppressor activity. J. Exp. Med. 190: 1375-1382.

34. Mitchell RA, Liao H, Chesney J, et al. (2002) Macrophage migration inhibitory factor (MIF) sustains macrophage proinflammatory function by inhibiting p53: regulatory role in the innate immune response. Proc. Natl. Acad. Sci. USA 99: 345-350.

35. Carter BD, Kaltschmidt C, Kaltschmidt B, et al. (1996) Selective activation of NF-kB by nerve growth factor through the neurotrophin receptor $\mathrm{p} 75$. Science 272: 542-545.

36. Syroid DE, Maycox PR, Burrola PG, et al. (1996) Cell death in the Schwann cell lineage and its regulation by neuregulin. Proc. Natl. Acad. Sci. USA 93: 9229-9234.

37. You S, Petrov T, Chung PH, Gordon T. (1997) The expression of the low affinity nerve growth factor receptor in long-term denervated Schwann cells. Glia 20: 87-100. 